\title{
Breakdown Behavior of 40-nm PD-SOI NMOS Device Considering STI-Induced Mechanical Stress Effect
}

\author{
V. C. Su, I. S. Lin, J. B. Kuo, Fellow, IEEE, G. S. Lin, D. Chen, C. S. Yeh, C. T. Tsai, and M. Ma
}

\begin{abstract}
This letter reports the shallow-trench-isolation (STI)-induced mechanical-stress-related breakdown behavior of the 40-nm PD-SOI NMOS device. As verified by the experimentally measured data and the 2-D simulation results, breakdown occurs at a higher drain voltage for the device with a smaller S/D length of $0.17 \mu \mathrm{m}$ due to the weaker function of the parasitic bipolar device, which is offset by the stronger impact ionization in the post-pinchoff region coming from the bandgap narrowing generated by the STI-induced mechanical stress.
\end{abstract}

Index Terms-CMOSFETs, silicon on insulator technology.

\section{INTRODUCTION}

$\mathbf{M}$ ECHANICAL stress induced by STI may affect the performance of CMOS devices [1], [2]. Mechanical stress may change work function, effective mass, carrier mobility, and carrier lifetime [3], [4]. For nanometer PD-SOI CMOS devices, the S/D region may be very small; the influence of the STI-induced mechanical stress cannot be overlooked. Recently, STI-induced mechanical stress effects on the kink behaviors of the PD-SOI NMOS devices have been reported [5], [6]. In this letter, STI-induced mechanical-stress-related breakdown behavior of the 40-nm PD-SOI NMOS device is reported. In the following sections, the STI-induced mechanical stress is described first, followed by STI-induced breakdown, discussion, and conclusion.

The 40-nm PD-SOI NMOS test device, with its cross section being shown in Fig. 1, has a 70-nm thin film doped with a p-type density of $3 \times 10^{18} \mathrm{~cm}^{-3}$ above a buried oxide of $145 \mathrm{~nm}$, under a gate oxide of $1.5 \mathrm{~nm}$. STI has been adopted. The channel width of the device is $1 \mu \mathrm{m}$. Two cases with S/D lengths of 1.7 and $0.17 \mu \mathrm{m}$, respectively, have been designed for this letter. Experimental measurements of the test device and 2-D simulation considering mechanical stress effects [7] have been used to carry out the study.

Manuscript received December 13, 2007; revised February 28, 2008. This work was supported under a research grant from Semiconductor Research Corporation. The review of this letter was arranged by Editor M. Ostling.

V. C. Su, I. S. Lin, and J. B. Kuo are with the Department of Electrical Engineering, National Taiwan University, Taipei 106, Taiwan, R.O.C. (e-mail: jbkuo@cc.ee.ntu.edu.tw).

G. S. Lin, D. Chen, C. S. Yeh, C. T. Tsai, and M. Ma are with United Microelectronics Corporation, Hsinchu 300, Taiwan, R.O.C.

Color versions of one or more of the figures in this letter are available online at http://ieeexplore.iee.org.

Digital Object Identifier 10.1109/LED.2008.922971
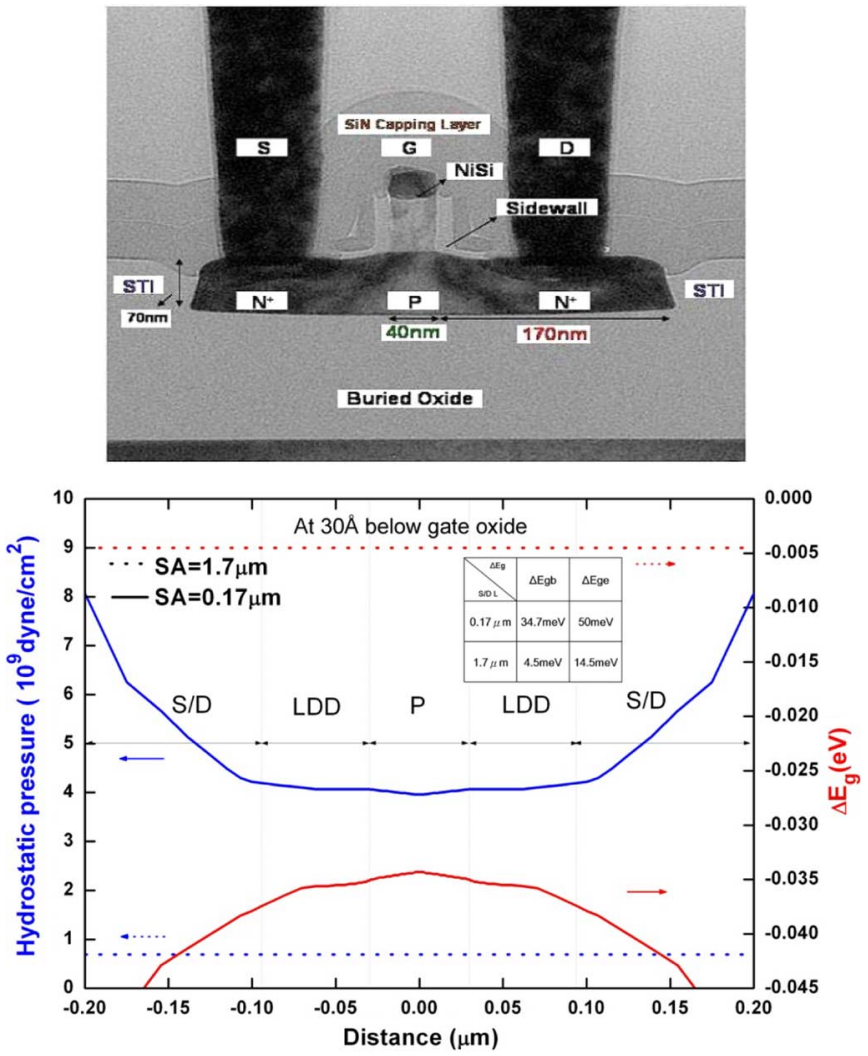

Fig. 1. (a) TEM cross section of the 40-nm PD-SOI NMOS device under study. (b) Mechanical stress and bandgap narrowing distribution at $3 \mathrm{~nm}$ below the gate oxide in the lateral direction of the PD device with S/D lengths of 0.17 and $1.7 \mu \mathrm{m}$.

In an SOI MOS device, the internal mechanical stress is caused during the formation of the STI structure. Fig. 1(b) shows the hydrostatic pressure and the associated bandgap narrowing distribution at $3 \mathrm{~nm}$ below the gate oxide interface in the lateral direction of the 40-nm PD-SOI NMOS device with S/D lengths of 0.17 and $1.7 \mu \mathrm{m}$ based on process simulation results [8]. As induced by STI, for the S/D length of $0.17 \mu \mathrm{m}$, the mechanical stress in terms of hydrostatic pressure is large in the S/D region near STI and decreases as the distance becomes farther away from it. The corresponding bandgap narrowing, which is correlated to the mechanical stress, has a dramatic impact on the breakdown behavior of the 40-nm PD-SOI NMOS device. 


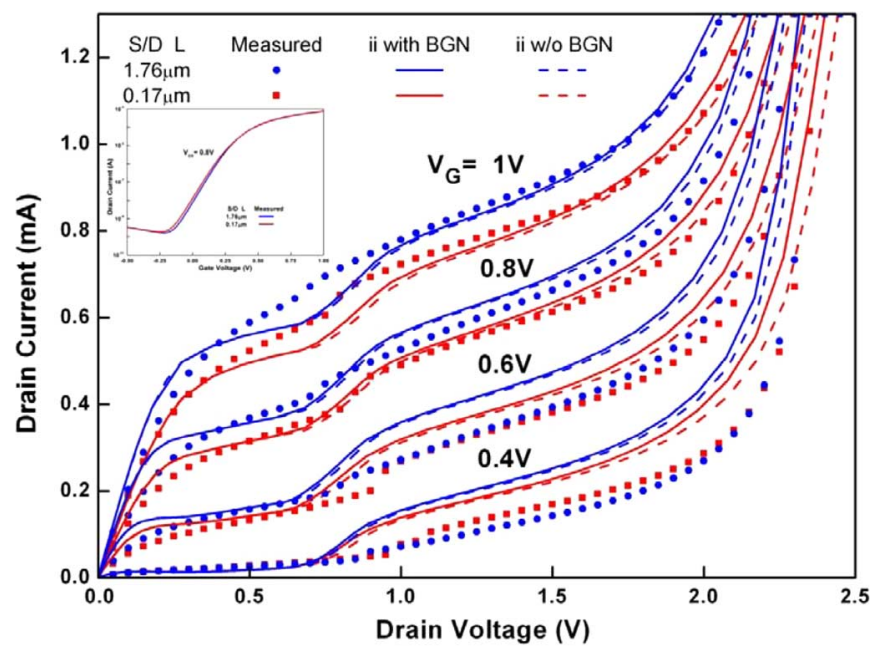

Fig. 2. $I_{D}$ versus $V_{D}$ of the 40-nm PD-SOI NMOS device with S/D lengths of 1.7 and $0.17 \mu \mathrm{m}$, based on the measured data and 2-D simulation results, with the inlet figure being the measured subthreshold figure.

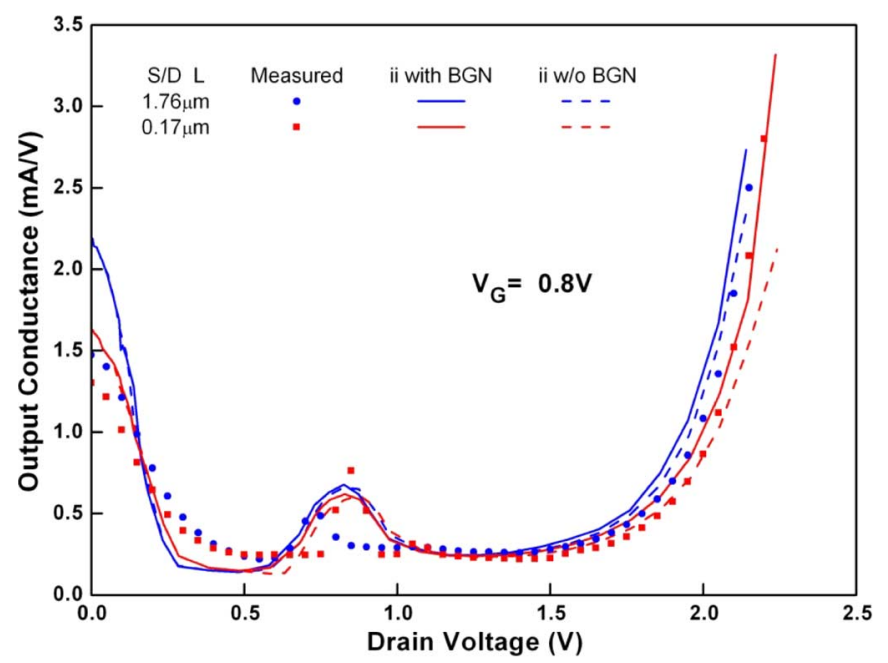

Fig. 3. Output conductance versus $V_{D}$ of the 40-nm PD-SOI NMOS device with S/D lengths of $L=1.7$ and $0.17 \mu \mathrm{m}$, biased at $V_{G}=0.8 \mathrm{~V}$, based on the measured data and 2-D simulation results.

\section{STI-INDUCED BREAKDOWN BEHAVIOR AND DISCUSSION}

Fig. 2 shows $I_{D}$ versus $V_{D}$ of the 40-nm PD-SOI NMOS device with S/D lengths of 1.7 and $0.17 \mu \mathrm{m}$, biased with its body floating, based on the measured data and 2-D device simulation results. Also shown in the inlet of the figure is the measured subthreshold figure. With the S/D length of $0.17 \mu \mathrm{m}$, the breakdown occurs at a larger $V_{D}$ as compared to the case with the S/D length of $1.7 \mu \mathrm{m}$. Fig. 3 shows the related output conductance versus $V_{D}$ characteristics of the PD device biased at $V_{G}=0.8 \mathrm{~V}$. As confirmed by the smaller output conductance, with the shorter S/D length of $0.17 \mu \mathrm{m}$, breakdown occurs at a larger $V_{D}$, which is due to the higher bandgap narrowing effects from the larger mechanical stress on the parasitic bipolar device. The trends predicted by the 2-D device simulation are strongly correlated to the experimentally measured data, as shown in the figures.

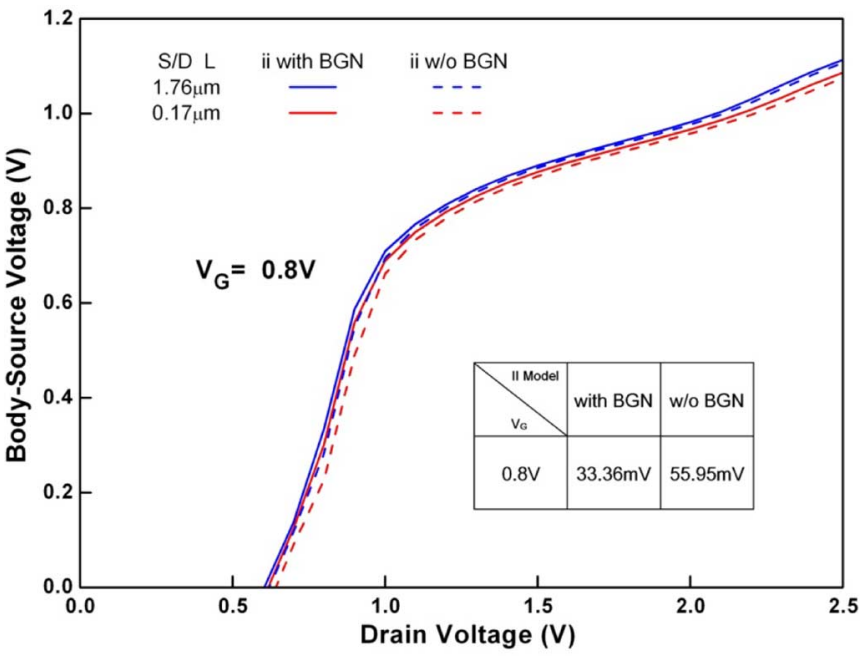

Fig. 4. Body-source voltage versus $V_{D}$ of the 40-nm PD-SOI NMOS device with S/D lengths of $L=1.7$ and $0.17 \mu \mathrm{m}$, biased at $0.8 \mathrm{~V}$, based on the 2-D simulation results.

From the current conduction mechanism inside the device [9], both the parasitic bipolar device and the post-pinchoff impact ionization determine the breakdown behavior. With a smaller S/D length of $0.17 \mu \mathrm{m}$, in the post-pinchoff region, the impact ionization is stronger because it is closely related to the bandgap [10]. On the other hand, the vitality of the parasitic bipolar device is also determined by the bandgap narrowing factor. Fig. 4 shows the body-source voltage, which is the base-emitter voltage $\left(V_{\mathrm{BE}}\right)$ of the parasitic bipolar device in the thin film, of the 40-nm PD-SOI NMOS device with S/D lengths of $L=1.7$ and $0.17 \mu \mathrm{m}$, biased at $V_{G}=0.8 \mathrm{~V}$, based on the 2-D simulation results. As shown in the figure, with a smaller S/D length of $0.17 \mu \mathrm{m}$, the body-source voltage is smaller at a larger $V_{D}$, which indicates the weaker function of the parasitic bipolar device. The less strong turn-on of the parasitic bipolar device at a high drain voltage for the $0.17-\mu \mathrm{m}$ case could be understood from the current-gain $\beta$ formula as a function of the difference in the bandgap narrowing between the base and the emitter [10]

$$
\beta \alpha \exp \left(\Delta E_{\mathrm{gb}}-\Delta E_{\mathrm{ge}} / k T\right)
$$

where $\Delta E_{\mathrm{gb}} / \Delta E_{\mathrm{ge}}$ is the bandgap narrowing in the base/emitter (the source), $k$ is the Boltzmann constant, and $T$ is the temperature in kelvin. Due to the higher mechanical stress from STI, the bandgap narrowing in the emitter (source) $\Delta E_{\mathrm{ge}}$ is larger than that in the base (the channel) region $\Delta E_{\mathrm{gb}}$. Therefore, the vitality of the parasitic bipolar device in the thin film of the device with a shorter S/D length of $0.17 \mu \mathrm{m}$ becomes less strong as indicated by the smaller current gain. Because the bandgap-narrowing-related bipolar function is the dominant factor in determining the breakdown behavior compared to the bandgap-related impact ionization in the postpinchoff region, the breakdown voltage becomes higher for the case with a smaller S/D length of $0.17 \mu \mathrm{m}$ due to a higher STI-induced mechanical stress. Fig. 5 shows the breakdown voltage versus the gate voltage of the 40-nm PD-SOI NMOS device with S/D lengths of $L=1.7,0.17$, and $0.017 \mu \mathrm{m}$, based 


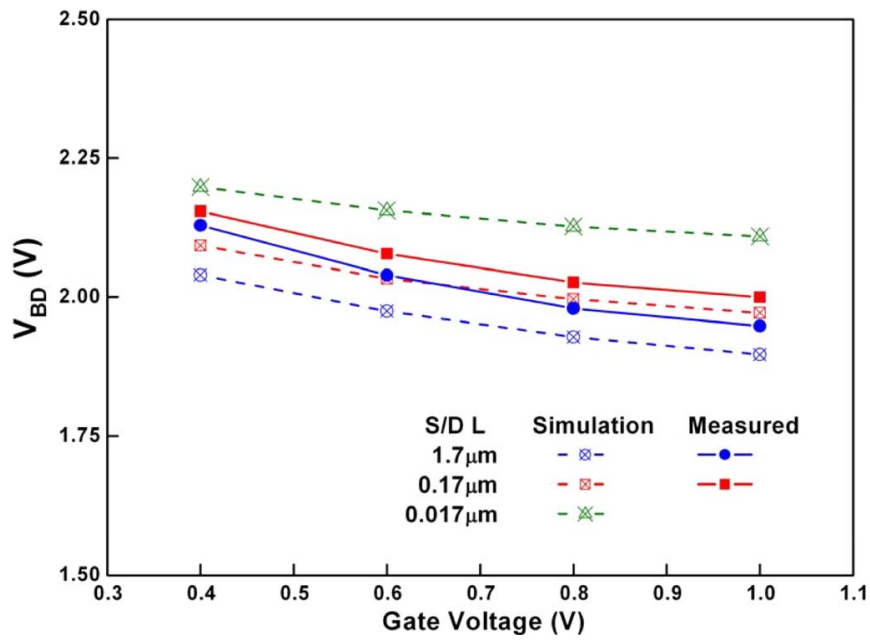

Fig. 5. Breakdown voltage versus gate voltage of the 40-nm PD-SOI NMOS device with S/D lengths of $L=1.7,0.17$, and $0.07 \mu \mathrm{m}$, based on the measured data and the 2-D simulation results.

on the measured data and the 2-D simulation results. From the figure, the 2-D simulation results based on the mechanicalstress bandgap narrowing are correlated to the measured data. As shown in the figure, a smaller gate voltage leads to a larger breakdown voltage due to the extra degradation of the parasitic BJT function due to the high injection effect coming from the increased impact ionization in the larger post-saturation region.

\section{CONCLUSION}

This letter reports the STI-induced mechanical-stress-related breakdown behavior of the 40-nm PD-SOI NMOS device. As verified by the experimentally measured data and the 2-D simulation results, breakdown occurs at a higher drain voltage for the device with a smaller S/D length of $0.17 \mu \mathrm{m}$ due to the weaker function of the parasitic bipolar device, which is offset by the stronger impact ionization in the post-pinchoff region coming from the bandgap narrowing generated by STI-induced mechanical stress.

\section{REFERENCES}

[1] R. A. Bianchi, G. Bouche, and O. Roux-dit-Buisson, "Accurate modeling of trench isolation induced mechanical stress effects on MOSFET electrical performance," in IEDM Tech. Dig., 2002, pp. 117-120.

[2] C. Gallon, G. Reimbold, G. Ghibaudo, R. A. Bianchi, R. Gwoziecki, S. Orain, E. Robilliart, C. Raynaud, and H. Dansas, "Electrical analysis of mechanical stress induced by STI in short MOSFETs using externally applied stress," IEEE Trans. Electron Devices, vol. 51, no. 8, pp. 1254-1261, Aug. 2004.

[3] J. Pretet, D. Ioannou, N. Subba, S. Cristoloveanu, W. Maszara, and C. Raynaud, "Narrow-channel effects and their impact on the static and floating-body characteristics of STI- and LOCOS-isolated SOI MOSFETs," Solid State Electron., vol. 46, no. 11, pp. 1699-1707, Nov. 2002.

[4] J. Pretet, N. Subba, D. Ioannou, S. Cristoloveanu, W. Maszara, and C. Raynaud, "Reduced floating body effects in narrow channel SOI MOSFETs," IEEE Electron Device Lett., vol. 23, no. 1, pp. 55-57, Jan. 2002.

[5] I. Lin, V. Su, J. B. Kuo, M. Ma, C. T. Tsai, C. S. Yeh, and D. Chen, "STI mechanical-stress induced subthreshold kink effect of $40 \mathrm{~nm}$ PD SOI NMOS device," in Proc. Dig. ISDRS, Dec. 2007, pp. 1-2.

[6] I. S. Lin, V. C. Su, J. B. Kuo, D. Chen, C. S. Yeh, C. T. Tsai, and M. Ma, "STI-induced mechanical stress-related kink effect of $40 \mathrm{~nm}$ PD SOI NMOS devices," in Proc. EUROSOI Dig., 2008, pp. 81-82.

[7] Taurus Medici User Guide, Synopsys Inc., Mountain View, CA, Oct. 2005.

[8] Taurus TSUPREM-4 User Guide, Synopsys Inc., Mountain View, CA, Oct. 2005.

[9] Y. G. Chen, S. Y. Ma, J. B. Kuo, and R. W. Dutton, "An analytical drain current model for short-channel FD ultrathin SOI NMOS devices," Solid State Electron., vol. 38, no. 12, pp. 2051-2057, Dec. 1995.

[10] S. Selberherr, Analysis and Simulation of Semiconductor Devices. New York: Springer-Verlag, 1984.

[11] J. B. Kuo et al., "Analytical bandgap-narrowing-related current-gain model for BJT devices operating at $77 \mathrm{~K}$," Solid State Electron., vol. 35, no. 6, pp. 785-790, Jun. 1992. 